\title{
Clinical significance of focal ß-amyloid deposition measured by ${ }^{18} \mathrm{~F}$-flutemetamol PET
}

Si Eun Kim ${ }^{1,2}$, Byungju Lee 3 , Seongbeom Park ${ }^{1,4,5}$, Soo Hyun Cho ${ }^{1,6}$, Seung Joo Kim ${ }^{1,7}$, Yeshin Kim ${ }^{8}$, Hyemin Jang ${ }^{1,4,5}$, Jee Hyang Jeong ${ }^{9}$, Soo Jin Yoon ${ }^{10}$, Kyung Won Park ${ }^{11}$, Eun-Joo Kim ${ }^{12}$, Na Yeon Jung ${ }^{13}$, Bora Yoon ${ }^{14}$, Jae-Won Jang ${ }^{8}$, Jin Yong Hong ${ }^{15}$, Jihye Hwang ${ }^{16}$, Duk L. Na ${ }^{1,4,5,17,18}$, Sang Won Seo ${ }^{1,4,5,19,20}$, Seong Hye $\mathrm{Choi}^{21^{*}}$ and Hee Jin Kim ${ }^{1,4,5^{*}}$

\begin{abstract}
Background: Although amyloid PET of typical Alzheimer's disease (AD) shows diffuse B-amyloid (Aß) deposition, some patients show focal deposition. The clinical significance of this focal $A B$ is not well understood. We examined the clinical significance of focal $A B$ deposition in terms of cognition as well as $A B$ and tau cerebrospinal fluid (CSF) levels. We further evaluated the order of $A ß$ accumulation by visual assessment.
\end{abstract}

Methods: We included 310 subjects (125 cognitively unimpaired, 125 mild cognitive impairment, and 60 AD dementia) from 9 referral centers. All patients underwent neuropsychological tests and ${ }^{18} \mathrm{~F}$-flutemetamol (FMM) PET. Seventy-seven patients underwent CSF analysis. Each FMM scan was visually assessed in 10 regions (frontal, precuneus and posterior cingulate, lateral temporal, parietal, and striatum of each hemisphere) and was classified into three groups: No-FMM, Focal-FMM (FMM uptake in 1-9 regions), and Diffuse-FMM (FMM uptake in all 10 regions).

Results: 53/310 (17.1\%) subjects were classified as Focal-FMM. The cognitive level of the Focal-FMM group was better than that of Diffuse-FMM group and worse than that of No-FMM group. Among the Focal-FMM group, those who had FMM uptake to a larger extent or in the striatum had worse cognitive levels. Compared to the DiffuseFMM group, the Focal-FMM group had a less AD-like CSF profile (increased Aß42 and decreased t-tau, t-tau/Aß42). Among the Focal-FMM group, $A ß$ deposition was most frequently observed in the frontal (62.3\%) and least frequently observed in the striatum (43.4\%) and temporal (39.6\%) regions.

Conclusions: We suggest that focal $A B$ deposition is an intermediate stage between no $A B$ and diffuse $A B$ deposition. Furthermore, among patients with focal $A B$ deposition, those who have $A B$ to a larger extent and striatal involvement show clinical features close to diffuse $A ß$ deposition. Further longitudinal studies are needed to evaluate the disease progression of patients with focal $A ß$ deposition.

Keywords: ß-amyloid, ${ }^{18}$ F-flutemetamol PET, Alzheimer's disease, Cognition, Cerebrospinal fluid

\footnotetext{
*Correspondence: seonghye@inha.ac.kr; evekhj@gmail.com

${ }^{21}$ Department of Neurology, Inha University School of Medicine, Incheon, Korea

${ }^{1}$ Department of Neurology, Samsung Medical Center, Sungkyunkwan

University School of Medicine, 50 Ilwon-dong, Gangnam-ku, Seoul 135-710,

Republic of Korea

Full list of author information is available at the end of the article
}

(C) The Author(s). 2020 Open Access This article is distributed under the terms of the Creative Commons Attribution 4.0 International License (http://creativecommons.org/licenses/by/4.0/), which permits unrestricted use, distribution, and reproduction in any medium, provided you give appropriate credit to the original author(s) and the source, provide a link to the Creative Commons license, and indicate if changes were made. The Creative Commons Public Domain Dedication waiver (http://creativecommons.org/publicdomain/zero/1.0/) applies to the data made available in this article, unless otherwise stated. 


\section{Background}

Alzheimer's disease (AD), the most common cause of dementia, is characterized by $ß$-amyloid $(A ß)$ and tau accumulation in the brain [1]. Aß accumulation starts approximately 10 to 20 years before dementia symptoms begin. Thus, detecting the presence of $A ß$ is essential for early diagnosis of $\mathrm{AD}$. With recent advances in detecting $A ß$ in vivo, the use of an $A ß$ biomarker is clinically available through PET imaging or CSF analysis [2, 3].

Although amyloid PET of typical AD shows diffuse Aß deposition, some patients show focal $A ß$ deposition, the clinical significance of which is not well defined. Previous studies lack pathological examination results of patients with focal $\mathrm{A} ß$ deposition on PET imaging. Brain $\mathrm{A} ß$ burden on PET imaging may be quantitatively measured in the research field using standardized uptake value ratios (SUVR) and studies showed that higher SUVR is correlated with poor clinical outcome [4-6]. Meanwhile, in clinical practice, interpretation of amyloid PET relies on dichotomous visual rating (positive or negative). According to visual interpretation guidelines of PET images such as ${ }^{18} \mathrm{~F}$-florbetaben or ${ }^{18} \mathrm{~F}$-flutemetamol (FMM), if any of the brain regions (frontal, parietal, precuneus/posterior cingulate (PPC), lateral temporal lobes, and striatum) is positive in either hemisphere, the scan is considered to be positive $[7,8]$. However, since $\mathrm{A} ß$ deposition is a gradual process [9], a dichotomous visual rating may be misleading. Identifying the clinical significance of participants in the gray zone may help manage patients in clinical practice. Thus, this particular group needs to be well characterized.

In this study, we hypothesized that patients showing focal $A ß$ deposition have unique clinical characteristics. We examined patients showing focal Aß deposition on FMM PET scan. We compared cognition and CSF AD biomarkers between patients with No-, Focal-, and Diffuse-FMM uptake. We also aimed to assess whether the extent and region of focal FMM uptake are related to cognition. We further evaluated the order of $A ß$ accumulation by visual assessment.

\section{Methods}

\section{Participants}

We recruited 310 patients with cognitively unimpaired (CU; $n=125$ ), mild cognitive impairment (MCI; $n=$ 125 ), and AD dementia (ADD, $n=60$ ) who underwent FMM PET between June 2015 and December 2017. The $\mathrm{CU}$ individuals had normal age-, sex-, and educationadjusted performance on standardized cognitive tests [10]. The participants with MCI met the criteria proposed by Petersen et al. [11]: (1) subjective memory complaints, (2) relatively normal performance in other cognitive domains, (3) normal activities of daily living (ADL), (4) objective memory impairment below - 1.5 SD on either verbal or visual memory tests, and (5) not demented. The ADD patients met the criteria for dementia by the Diagnostic and Statistical Manual of Mental Disorders 4th Edition, Text Revision (DSM-IV-TR) [12] and were diagnosed with probable ADD according to the NIA-AA core clinical criteria [1]. The patients were recruited from 9 referral hospitals in South Korea (175 from Samsung Medical Center, 135 from Validation Cohort of Korean Brain Aging Study for the Early Diagnosis and Prediction of AD (KBASE-V) [13]). All participants underwent neurologic examination, neuropsychological test, and Apolipoprotein E (APOE) genotyping. We screened blood tests including a complete blood count, blood chemistry, thyroid function, vitamin B12, folate, and syphilis serology and excluded participants with abnormal findings that could affect their cognition. Participants with previous or current neurological or psychiatric diseases such as brain tumors, encephalitis, epilepsy, and depressive disorders that could affect cognitive function were also excluded. On MRI, patients with structural lesions such as hydrocephalus, brain tumors, or traumatic brain injuries were also excluded. The Institutional Review Boards approved this study at all participating centers. We obtained written, informed consent from patients and caregivers.

\section{${ }^{18} \mathrm{~F}$-flutemetamol PET acquisition and analysis}

We performed FMM PET scans using a Discovery 600 PET/CT scanner (GE), Discovery 690 PET/CT scanner (GE), Discovery STE PET/CT scanner (GE), Biography MCT PET/CT scanner (Siemens) or Gemini TF PET/CT scanner (Philips) on a total number of 310 participants as described in a previous study [13]. The participants underwent a $20 \mathrm{~min}$ PET scan $(4 \times 5 \mathrm{~min}$ dynamic frames $)$ starting at 90 min after intravenous injection of $185 \mathrm{MBq}$ $\pm 10 \%$ of ${ }^{18} \mathrm{~F}$-flutemetamol. Low-dose computed tomography was utilized for attenuation correction before scans. We reconstructed the images with the Ordered Subsets Expectation Maximization algorithm using 4 iterations and 16 subsets.

\section{Blinded visual interpretation}

Visual interpretation of the FMM PET images was performed by two blinded neurologists (referred to as "readers") who successfully completed the manufacturing company's electronic training program. Visual interpretation of FMM PET images was performed by systematic review of ten brain regions (frontal, parietal, PPC, striatum, and lateral temporal lobes in each hemisphere) [14]. For each region, the readers used dichotomous assessment in classifying images as either normal or abnormal in a rainbow color scale anchored to the pons. We defined each region to be abnormal when there was increased cortical gray matter signal (above 50-60\% peak intensity) 
and/or reduced (or absent) gray-white matter contrast [15]. Inter-reader agreement of interpretation of FMM PET was excellent (kappa score $=0.94)$.

We classified patients into three groups. No-FMM (no FMM uptake in any region), Focal-FMM (FMM uptake in 1-9 regions), and Diffuse-FMM (FMM uptake in all 10 regions). Examples of FMM PET images are shown in Additional file 1: Figure S1.

\section{Cerebrospinal fluid collection, processing, and analysis} CSF sampling was performed in 77 participants (49 CU, $16 \mathrm{MCI}$, and $12 \mathrm{ADD}$ ) by procedures as previously described [13]. We obtained CSF in $15 \mathrm{~mL}$ polypropylene tubes (Falcon, Corning Science, NY, USA) and centrifuged at $2000 \times g$ for $10 \mathrm{~min}$ at room temperature (RT). Approximately $10 \mathrm{cc}$ of the CSF supernatant was frozen and transferred to the laboratory at Inha University. For measuring CSF biomarkers, the CSF was thawed and gently extracted into pipettes with polypropylene tips. A total of $0.4 \mathrm{~mL}$ aliquots of CSF was frozen in polypropylene tubes (Sarstedt AG \& Co., Nümbrecht, Germany) and stored at $-80^{\circ} \mathrm{C}$ until analysis. We measured the level of CSF Aß 42, total tau (t-tau), and phosphorylated tau (p-tau) using the multiplex xMAP Luminex platform with INNO-BIA AlzBio3 kits. AlzBio3 kits (Fujirebio Europe, Ghent, Belgium) contained capture monoclonal antibodies for $A ß 42$, $t$-tau, and $\mathrm{p}$-tau, which linked to two aqueous quality controls (a-QC) with pre-defined concentration ranges for the three biomarkers. The procedure is described elsewhere $[16,17]$. To reduce the effects of sources of variability on the results [18], CSF analysis was followed by the manufacturer's instructions and standard of procedures that were previously described $[19,20]$.

\section{Neuropsychological evaluation}

All participants underwent a standardized neuropsychological battery called the Korean version of the Consortium to Establish a Registry for Alzheimer's Disease Assessment Packet (CERAD-K) [21] or Seoul Neuropsychological Screening Battery (SNSB) [22], which consisted of tests of language, visuospatial, memory, and frontal/executive functions.

Tests in CERAD-K included the Korean version of the Boston Naming Test (K-BNT) to assess language function; constructional praxis (copying figures) to assess visuospatial function; 10 word list recall (20-min delayed recall) and constructional recall (20-min delayed figure recall) to assess verbal and visual memory, respectively; Controlled Oral Word Association Test (COWAT: animal naming) and Stroop Test (color reading) to assess frontal/executive function; and the Mini-Mental State Examination (MMSE) to assess global cognitive function.
Tests in SNSB included the K-BNT to assess language function; Rey-Osterrieth Complex Figure Test (RCFT: copying) to assess visuospatial function; Seoul Verbal Learning Test (20-min delayed recall) and RCFT (20min delayed recall) to assess verbal and visual memory, respectively; COWAT (animal naming) and the Stroop Test (color reading) to assess frontal-executive function; and the MMSE to assess global cognitive function. [23]

Tests were conducted by experienced staff and supervised by board-certified neuropsychologists. The norms for each test were based on 1987 normal Korean participants (for CERAD-K) or 1067 normal Korean participants (for SNSB). In the analyses, we used the $z$-scores of each test, which were based on the mean and standard deviation of each measure in the age- and education-matched norms.

\section{Statistical analysis}

For descriptive statistics, we used the chi-square test and analysis of variance (ANOVA) followed by Bonferroni's post hoc analyses.

To compare the cognitive profile of the three groups (No-FMM, Focal-FMM, and Diffuse-FMM group), we used ANOVA followed by Bonferroni's post hoc analyses. When we compared the cognitive profile of the Regional-FMM group with the No-FMM or the Diffuse-FMM group, we used ANOVA followed by Bonferroni's correction for 30 multiple tests ( 5 regions and 6 cognitive tests). To evaluate the association between cognition and number of FMM uptake regions, we used linear regression analyses.

For comparison of the CSF profile of the three groups, we used analysis of covariance (ANCOVA) followed by Bonferroni's post hoc analyses after controlling for age. To evaluate the association between CSF profile and number of FMM uptake regions, linear regression analyses were used after adjusting for age. All statistical tests were performed using MedCalc (MedCalc Software version 19, Ostend, Belgium).

To determine the spreading order of FMM uptake, we assumed that regions with earlier appearance of pathology would show abnormal FMM uptake in a greater number of participants, as suggested by previous studies $[24,25]$. The different frequency of regional involvement was assessed using a bootstrapping method with 1000 re-samples in R v3.1.3 (Institute for Statistics and Mathematics, Vienna, Austria; www.R-project.org), which derived the estimates of 95\% confidence intervals and standard error. We calculated asymptotic $p$ values and corrected for multiple comparisons with Bonferroni's method for all combinations of regional pairs.

\section{Results}

Characteristics of the participants

The demographic and clinical characteristics are presented in Table 1. Of all the participants, 17.1\% (53/310) 
Table 1 Demographic characteristics of participants

\begin{tabular}{|c|c|c|c|c|c|c|}
\hline & $\begin{array}{l}\text { No-FMM uptake } \\
(n=174)\end{array}$ & $\begin{array}{l}\text { Focal-FMM uptake } \\
(n=53)\end{array}$ & $\begin{array}{l}\text { Diffuse-FMM uptake } \\
(n=83)\end{array}$ & $\begin{array}{l}p \\
\text { No vs focal } \\
\text { uptake }\end{array}$ & $\begin{array}{l}p \\
\text { No vs diffuse } \\
\text { uptake }\end{array}$ & $\begin{array}{l}p \\
\text { Focal vs diffuse } \\
\text { uptake }\end{array}$ \\
\hline Age (mean $\pm S D)$ & $69.4 \pm 8.6$ & $73.5 \pm 7.9$ & $71.4 \pm 8.5$ & 0.008 & 0.253 & 0.494 \\
\hline Men (\%) & $73(42.0)$ & $15(28.3)$ & $37(44.6)$ & 0.075 & 0.692 & 0.058 \\
\hline Education, years (mean $\pm \mathrm{SD}$ ) & $10.7 \pm 5.0$ & $10.3 \pm 5.0$ & $11.0 \pm 4.5$ & 1.000 & 1.000 & 1.000 \\
\hline$A P O E$ \&4 carrier (\%) & 21/166 (12.7) & 23/53 (43.4) & $42 / 81(51.9)$ & $<0.001$ & $<0.001$ & 0.340 \\
\hline $\begin{array}{l}\text { Disease duration (months) } \\
\text { (Mean } \pm \text { SD) }\end{array}$ & $62.5 \pm 52.9$ & $50.3 \pm 44.5$ & $41.5 \pm 41.6$ & 0.455 & 0.009 & 1.000 \\
\hline \multicolumn{7}{|l|}{ Vascular risk factors } \\
\hline Hypertension (\%) & $80(46.0)$ & $23(43.4)$ & $29(34.9)$ & 0.742 & 0.095 & 0.324 \\
\hline Diabetes (\%) & $33(19.0)$ & $5(9.4)$ & $11(13.3)$ & 0.104 & 0.257 & 0.502 \\
\hline Hyperlipidemia (\%) & $57(32.8)$ & $18(34.0)$ & $11(13.3)$ & 0.871 & 0.001 & 0.004 \\
\hline Cognitive level & & & & $<0.001$ & $<0.001$ & $<0.001$ \\
\hline CU (\%) & $102(58.6)$ & $17(32.1)$ & $6(7.2)$ & & & \\
\hline $\mathrm{MCl}(\%)$ & $64(36.8)$ & $20(37.7)$ & $41(49.4)$ & & & \\
\hline ADD (\%) & $8(4.6)$ & $16(30.2)$ & $36(43.4)$ & & & \\
\hline
\end{tabular}

Abbreviations: $F M M{ }^{18} \mathrm{~F}$-flutemetamol, $A P O E \varepsilon 4$ Apolipoprotein $\varepsilon 4, C U$ cognitively unimpaired, $M C l$ mild cognitive impairment, $A D D$ Alzheimer's disease dementia

patients were classified into the Focal-FMM group. The proportion in the Focal-FMM group and the extent of Focal-FMM uptake differed according to cognitive level. $13.6 \%(17 / 125)$ of $\mathrm{CU}, 16.0 \%(20 / 125)$ of $\mathrm{MCI}$, and $26.7 \%(16 / 60)$ of ADD were classified into the FocalFMM group. Among the Focal-FMM group, the median number of uptake regions was $1.0(95 \% \mathrm{CI}=1.0-4.0)$ in $\mathrm{CU}, 3.5(95 \% \mathrm{CI}=2.0-5.8)$ in $\mathrm{MCI}$, and $6.0(95 \% \mathrm{CI}=$ 3.6-8.0) in ADD. The Focal-FMM group was older than the No-FMM group and had more $A P O E \& 4$ carriers compared to the No-FMM group. In addition, there were statistically significant differences in distribution of cognitive level across the groups.

\section{Cognitive profiles of Focal-FMM group}

Compared to the No-FMM group, the Focal-FMM group showed significantly lower performance in all cognitive domains except for visuospatial function. Compared to the Diffuse-FMM group, the Focal-FMM group showed better performance in verbal memory and visual memory functions. Global cognitive function (measured by MMSE) of the Focal-FMM group was better than the Diffuse-uptake group but worse than the No-FMM group (Table 2).

Among the Focal-FMM group, as the number of FMM uptake regions increased, $z$-scores decreased in all cognitive domains such as K-BNT $(\beta=-0.264, p=$ $0.004)$, visuospatial function $(\beta=-0.290, p=0.010)$, verbal memory $(\beta=-0.105, p=-0.105)$, visual memory $(\beta=-0.138, p=0.021)$, COWAT $(\beta=-0.162, p=0.004)$, Stroop Test $(\beta=-0.239, p=0.004)$, and MMSE $(\beta=-$ 0.306, $p=0.016$ ) (Fig. 1.).
We further divided Focal-FMM group into patients with less FMM uptake (1-5 regions involved) group and patients with more FMM uptake (6-9 regions involved) group. Focal-FMM group with 1-5 regions involved did not show significant difference compared to the NoFMM group, while it showed better performance in all cognitive domains except for language when compared to Diffuse-FMM group. Focal-FMM group with 6-9 regions involved showed worse performance in all cognitive domains compared to No-FMM group, while it did not show significant difference when compared to Diffuse-FMM group (Additional file 1: Table S1).

Then, we compared each regional Focal-FMM group with the Focal- or Diffuse-FMM group to evaluate the regional effects of focal FMM uptake on cognitive function. Compared to the Diffuse-FMM group, verbal memory scores were higher in the Focal-FMM group with frontal, lateral temporal, parietal, or PPC regional involvement whereas no difference was found in the Focal-FMM group with striatal involvement. Compared to the Diffuse-FMM group, visual memory scores were higher in the Focal-FMM group with frontal, lateral temporal, or parietal regional involvement whereas no difference was found in the Focal-FMM group with PPC or striatal involvement. Compared to the No-FMM group, verbal and visual memory scores were lower in the Focal-FMM group with PPC or striatal involvement (Table 2).

Comparisons of cognitive scores between the No-, Focal-, and Diffuse-FMM groups in each cognitive level (CU, MCI and ADD) are shown in Additional file 1: Table S2). Among CU individuals, the cognitive score 


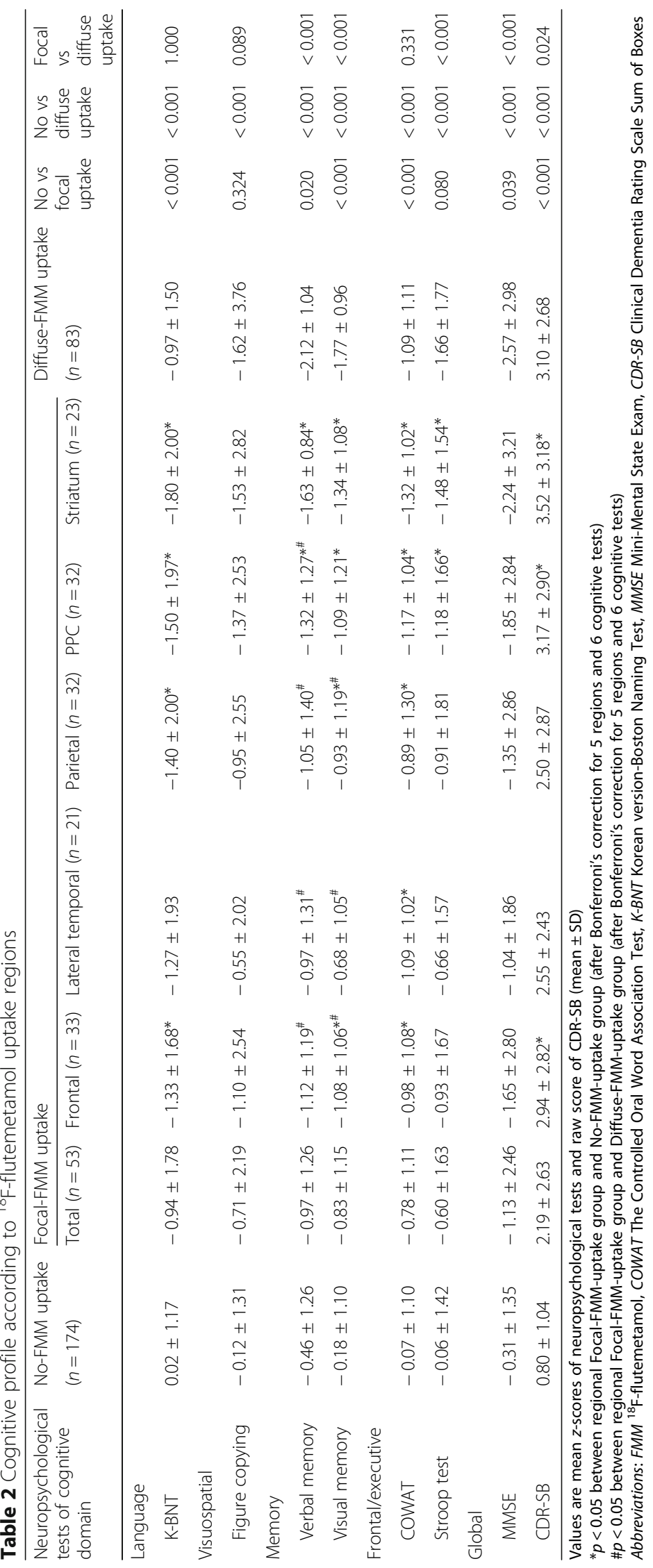



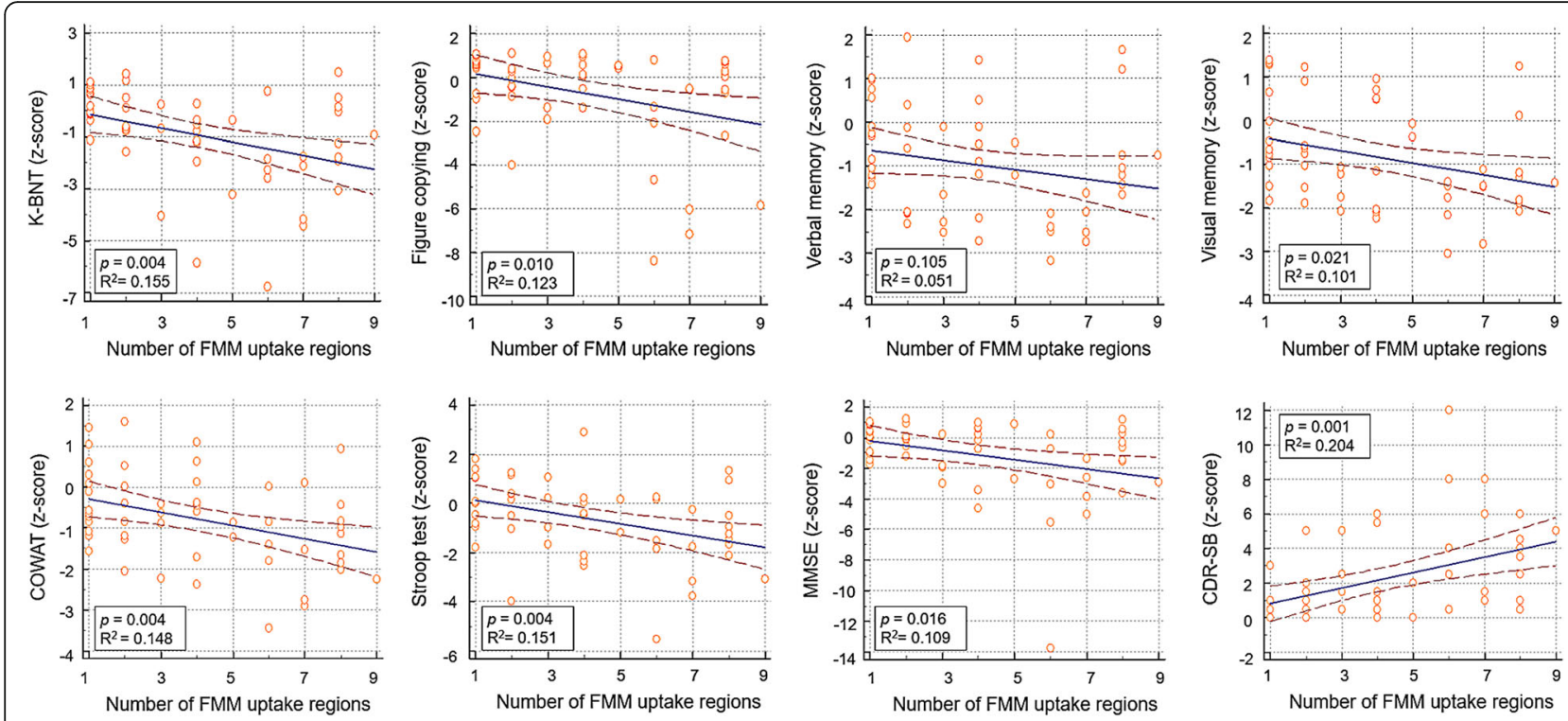

Fig. 1 Cognitive profile according to number of FMM uptake regions among the Focal-FMM group. Solid blue line is the regression line. Brown dotted lines indicate $95 \%$ confidence intervals. Abbreviations: FMM $={ }^{18} \mathrm{~F}$-flutemetamol; K-BNT = Korean version-Boston Naming Test; COWAT = The Controlled Oral Word Association Test; MMSE = Mini-Mental State Exam

did not differ among the No-, Focal-, and Diffuse-FMM groups. However, among MCI patients, the Focal-FMM group showed better performance in verbal and visual memory function, as well as in MMSE score, compared to the Diffuse-FMM group. Among ADD patients, Focal-FMM patients performed worse in language and frontal function compared to those of the No-FMM group and performed better in verbal memory than those of the Diffuse-FMM group. We provided a breakdown of cases by clinical designation and number of regions in Additional file 1: Table S3).

\section{CSF amyloid and tau level of focal-FMM group}

Levels of CSF AD biomarkers ( $A ß 42$, p-tau, t-tau, ptau/ $A ß 42$, and $t$-tau/ $A ß 42$ ) in No-, Focal-, and Diffuse-FMM groups are shown in Fig. 2. The FocalFMM group showed increased levels of CSF Aß 42 and decreased levels of CSF $t$-tau and $t$-tau/Aß 42, compared to the Diffuse-FMM group. However, there were no differences between Focal-FMM-uptake and No-FMMuptake groups except for p-tau level.

\section{Spreading order of FMM-uptake among focal-FMM- uptake group}

Among the Focal-FMM group, $A ß$ deposition was most frequently observed in the frontal $(62.3 \%, 95 \% \mathrm{CI}=$ 48.8-75.8) followed by PPC (60.4\%, 95\% CI $=46.8-74.0)$, parietal $(60.4 \%, 95 \% \mathrm{CI}=46.8-74.0)$, striatum $(43.4 \%$, 95\% CI $=29.6-57.2)$, and lateral temporal $(39.6 \%$, 95\% $\mathrm{CI}=26.0-53.2)$ regions (Fig. 3).

\section{Discussion}

In this study, we investigated the clinical significance of patients with Focal-FMM uptake, which consisted of $17.1 \%$ of all participants. Our major findings were as follow. First, cognitive function of patients with FocalFMM uptake was in the intermediate stage between patients with No- and Diffuse-FMM uptake. Among Focal-FMM patients, the larger extent and striatal involvement of FMM uptake was associated with worse cognition. Second, CSF AD biomarkers of Focal-FMM group were less AD-like compared to the Diffuse-FMM group. Finally, among the Focal-FMM group, FMM uptake was most frequently observed in the frontal and least observed in the striatum and lateral temporal regions. Taken together, our findings suggest that patients with Focal-FMM uptake have unique clinical characteristics. Furthermore, among patients with focal FMM uptake, those who have larger extent and striatal involvement of FMM showed clinical features close to diffuse $A ß$ uptake and, thus, might be considered as being in more advanced stage of AD.

We found that $17.1 \%$ of all participants had FocalFMM uptake. Focal-FMM consisted substantial portion of participants in each cognitive level: $13.6 \%$ (17/125) of UC, $16.0 \%$ (20/125) of MCI, and 26.7\% (16/60) of ADD. In clinical practice, interpretation of amyloid PET relies on visual assessment, which currently guides focal-FMM uptake to be read as positive for $A ß$. However, the clinical significance of focal $A ß$ uptake is not well understood. Characterizing participants in this gray zone may help better manage patients. 

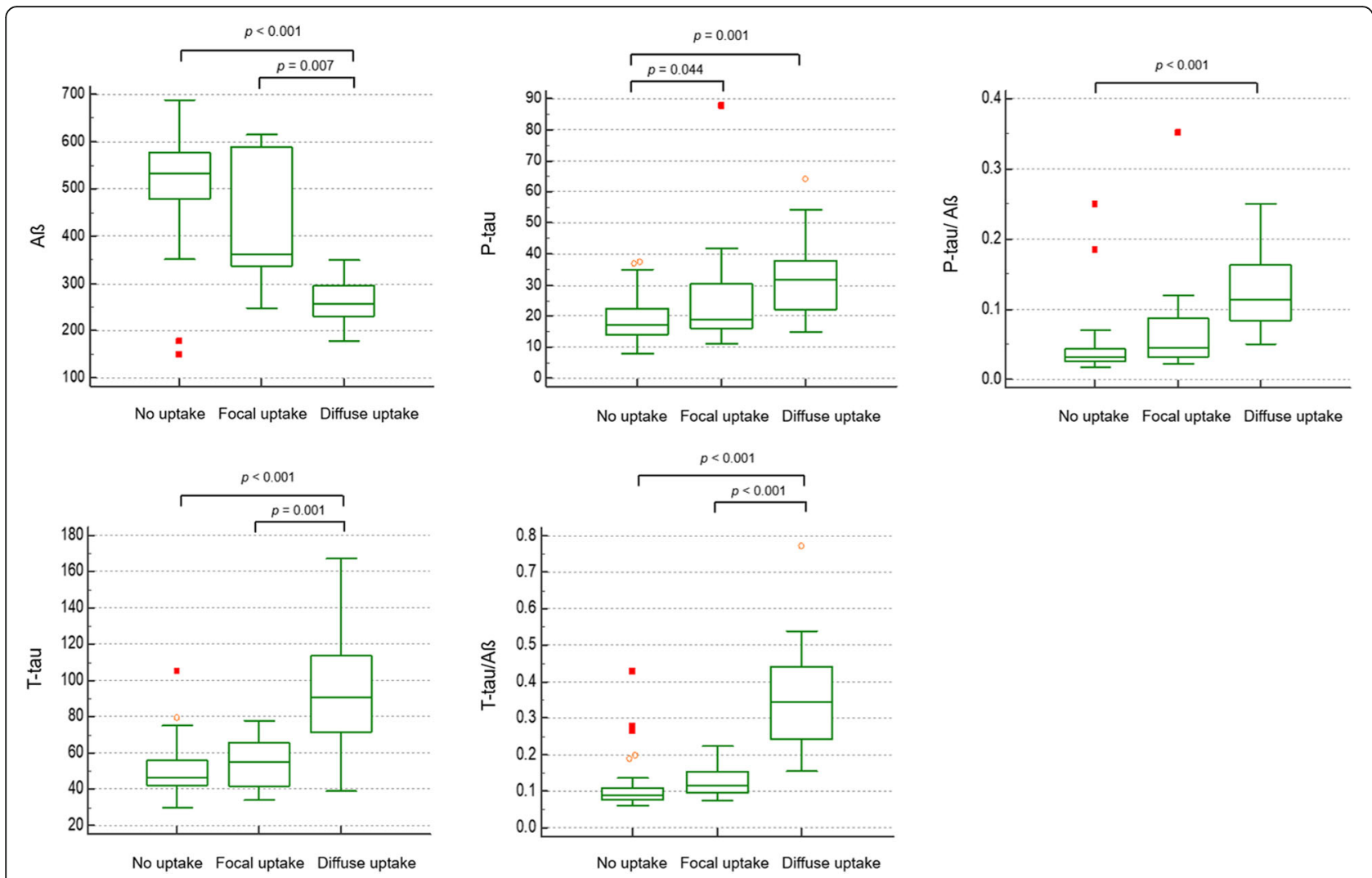

Fig. 2 Comparison of Alzheimer's disease biomarkers (Aß, P-tau, and T-tau) from cerebrospinal fluid among No-, Focal-, and Diffuse-FMM groups after adjusted for age. Box and whisker plots show medians, lower to upper quartile, and lines extending from minimum to maximum values. Abbreviations: FMM $={ }^{18}$ F-flutemetamol; P-tau $=$ phosphorylated tau; T-tau = total tau

Our first major finding was that cognitive function of patients with Focal-FMM was in the intermediate stage between patients with No- and Diffuse-FMM uptake. More importantly, the cognitive function differed according to the number and location of regions of focal FMM uptake. Among the Focal-FMM group, cognitive scores decreased with increasing number of FMM uptake regions. Our results are in line with previous studies using quantitative measurement of Aß burden [PIB [2628], ${ }^{18}$ F-florbetapir [29], or FMM [30] SUVR]. Subjects with higher PiB SUVR showed lower scores on episodic memory tests [27]. Higher FMM SUVR correlated with lower delayed memory index [30], and higher ${ }^{18}$ F-florbetapir SUVR correlated with lower MMSE scores [29]. Among the Focal-FMM group, those who had FMM uptake in the striatum had the worst cognitive scores. Although there have been numerous studies on the associations between quantitative $A ß$ deposition and cognition, no study has reported the association between visually assessed $A ß$ deposition and cognitive profiles. As quantitative analysis is not widely used in clinical practice, studies on visual assessment is valuable for clinicians. Our results suggest that when managing patients showing focal FMM uptake, clinicians should consider the number and location of regions with focal $A ß$ deposition.

Our second major finding was that patients with focal $A ß$ deposition on PET showed less AD-like CSF profiles compared to the Diffuse-FMM group. In addition, the increased number of FMM uptake regions significantly correlated with CSF biomarker levels toward a more AD-like pattern (increased Aß42 and decreased t-tau, ttau/ Aß42). Our results are consistent with previous studies showing negative correlation between CSF Aß42 levels and PET-based quantitative uptake of ${ }^{18} \mathrm{~F}$-florbetapir [31] or ${ }^{18}$ F-florbetaben [32].

Our third major finding was the order of $A ß$ accumulation. Among the Focal-FMM-group, $A ß$ deposition was most frequently observed in the frontal $(62.3 \%)$ followed by the PPC (60.4\%), parietal $(60.4 \%)$, and least frequently observed in the striatum (43.4\%) and lateral temporal (39.6\%) regions. Unlike the Thal stage of amyloid deposition [9], our data showed that striatal involvement preceded the involvement of lateral temporal region. However, our result generally reflects a downward spreading pattern of $A ß$, suggesting that $A ß$ deposits first in the cortex followed by subcortical structures [9]. 


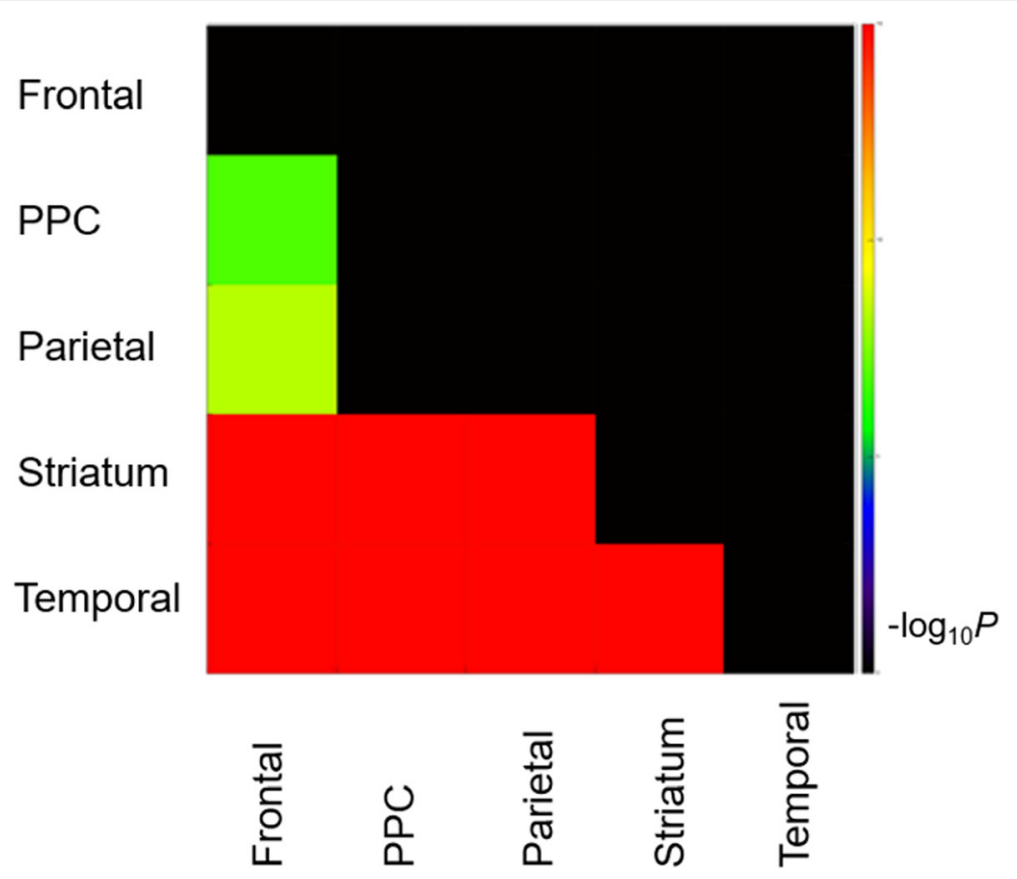

Fig. 3 Spreading order of FMM among Focal-FMM group ( $N=53)$. It shows the statistical significance in the comparison of the frequencies of FMM regional involvement between each pair of regions. The regional differences of uptake frequency display a stepwise pattern. Only the pairs of comparison passing Bonferroni's multiple comparisons are shown. Color bars represent logarithmic scale of $p$ value $\left(-\log _{10}\right)$. Abbreviation: $\mathrm{PPC}=$ precuneus/posterior cingulate

Furthermore, our data revealed that, among FocalFMM patients, those with subcortical $A ß$ involvement (striatum) showed lower cognition than those with cortical Aß involvement (frontal, lateral temporal, parietal, and PPC). Our findings are concordant with those of previous studies which found that subcortical $A ß$ involvement, especially striatal $A ß$, was related to worse cognitive performance and faster cognitive decline [33-35]. Patients with striatal involvement implies that they had higher Thal stage $(A ß$ phase 3$)$ and thus are more likely to have tau. Therefore, we suggest that even when $A ß$ involvement is focal, $A ß$ deposition in the striatum might be a sign of possible worse clinical outcome.

However, the present study has some limitations. First, our study used a cross-sectional design and, therefore, we do not know the cognitive trajectory of participants. Further longitudinal studies are warranted to evaluate the disease progression rate of the Focal-FMM group. Second, we lack pathological data on Focal-FMM patients. Amyloid PET-negative MCI or dementia patients in our data might have vascular, hippocampal sclerosis, or other pathologies as the main etiology for cognitive impairment. Further studies that could exclude non-AD pathologies are necessary. Nevertheless, the strength of our current study is that we have identified the clinical significance of focal $A ß$ deposition, which comprised a substantial portion of participants in each cognitive level.

\section{Conclusions}

In the current study, we found that focal $A ß$ deposition has unique clinical characteristics that differ from patients with no or diffuse $A ß$ deposition. We suggest that focal $A ß$ deposition should be considered as an intermediate stage between no $A ß$ and diffuse $A ß$ deposition. In addition, when managing patients showing focal $A B$ deposition, clinicians should consider the number and location of regions of focal $A ß$ deposition. Those with more regions involved, especially in the striatum, show clinical features close to diffuse $A ß$ deposition. Thus, cognitively unimpaired or MCI individuals with such signs might be more closely monitored for future cognitive decline. Further longitudinal studies are needed to evaluate the disease progression of patients with focal $A ß$ deposition.

\section{Supplementary information}

The online version of this article (https://doi.org/10.1186/s13195-019-0577-x) contains supplementary material, which is available to authorized users.

Additional file 1: Table S1. Cognitive profiles according to the number of 18F-flutemetamol uptake regions. Table S2. Cognitive profile of No-, Focal-, and Diffuse-FMM groups in each cognitive level. Table S3. Number of uptake regions in cognitively unimpaired, mild cognitive impairment, and Alzheimer's disease dementia. Figure S1. Examples of ${ }^{18} \mathrm{~F}$ - 
flutemetamol (FMM) PET scan in 'No-FMM', 'Focal-FMM' and 'DiffuseFMM' groups. Red arrows and dashed circles show FMM uptake, while white arrows and dashed circles indicate no FMM uptake. (DOCX 2813 kb)

\section{Abbreviations}

AD: Alzheimer's disease; ADD: AD dementia; Aß: B-amyloid; ANOVA: Analysis of variance; ANCOVA: Analysis of covariance; a-QC: Aqueous quality controls; CERAD-K: The Korean version of the Consortium to Establish a Registry for Alzheimer's Disease Assessment Packet; COWAT: Controlled Oral Word Association Test; CU: Cognitively unimpaired; CDR-SB: Clinical Dementia Rating Scale Sum of Boxes; DSM-IV-TR: Diagnostic and Statistical Manual of Mental Disorders 4th Edition, Text Revision; FMM: ${ }^{18}$ F-Flutemetamol; KBASEV: Korean Brain Aging Study for the Early Diagnosis and Prediction of AD; K BNT: Korean version-Boston Naming Test; MCl: Mild cognitive impairment: MMSE: Mini-Mental State Examination; PPC: Precuneus/posterior cingulate; $p$ tau: Phosphorylated tau; RCFT: Rey-Osterrieth Complex Figure Test: SUVR: Standardized uptake value ratios; SNSB: Seoul Neuropsychological Screening Battery; t-tau: Total tau

\section{Acknowledgements}

Not applicable.

\section{Authors' contribution}

SEK, SHC, and HJK contributed to the conceptualization of the study, analysis and interpretation of data, and drafting. BL and SBP contributed to the analyses of imaging data and preparation of the figures and provided technical support. SHC, SJK, YK, HJ, JHJ, SJY, KWP, EJK, NYJ, and BY contributed to the data collection and interpretation of data. JJ, JYH, JH, DLN, and SWS contributed to the analysis and interpretation of data. All authors read and approved the final manuscript.

\section{Funding}

This research was supported by the Original Technology Research Program for Brain Science through the National Research Foundation of Korea (NRF) funded by the Korean government (MSIP) (No. 2014M3C7A1064752); by the National Research Foundation of Korea (NRF) grant funded by the Korea government (MSIP) (NRF- 2018R1A1A3A04079255); and a grant of the Korean Health Technology R\&D Project, Ministry of Health \& Welfare, Republic of Korea (H118C1629 and H118C0335).

\section{Availability of data and materials}

The datasets used and/or analyzed during the current study are available from the corresponding author on reasonable request.

\section{Ethics approval and consent to participate}

The Institutional Review Boards approved this study at all participating centers. We obtained written, informed consent from patients and caregivers.

\section{Consent for publication}

Not applicable.

\section{Competing interests}

The authors declare that they have no competing interests.

\section{Author details}

'Department of Neurology, Samsung Medical Center, Sungkyunkwan University School of Medicine, 50 Ilwon-dong, Gangnam-ku, Seoul 135-710, Republic of Korea. ${ }^{2}$ Department of Neurology, Inje University College of Medicine, Haeundae Paik Hospital, Busan, Korea. ${ }^{3}$ Department of Neurology, Yuseong Geriatric Rehabilitation Hospital, Pohang, Korea. ${ }^{4}$ Samsung Alzheimer Research Center, Samsung Medical Center, Seoul, Korea. ${ }^{5}$ Neuroscience Center, Samsung Medical Center, Seoul, Korea. ${ }^{6}$ Department of Neurology, Chonnam National University Hospital, Chonnam National University Medical School, Gwangju, Korea. ${ }^{7}$ Department of Neurology, Gyeongsang National University School of Medicine and Gyeongsang National University Changwon Hospital, Changwon, Korea. ${ }^{8}$ Department of Neurology, Kangwon National University Hospital, Kangwon National University College of Medicine, Chuncheon, Korea. ${ }^{9}$ Department of Neurology, Ewha Womans University Mokdong Hospital, Ewha Womans
University School of Medicine, Seoul, Korea. ${ }^{10}$ Department of Neurology, Eulji University Hospital, Eulji University School of Medicine, Daejeon, Korea. ${ }^{11}$ Department of Neurology, Dong-A Medical Center, Dong-A University College of Medicine, Busan, Korea. ${ }^{12}$ Department of Neurology, Pusan National University Hospital, Pusan National University School of Medicine and Medical Research Institute, Busan, Korea. ${ }^{13}$ Department of Neurology, Pusan National University Yangsan Hospital, Pusan National University School of Medicine and Research Institute for Convergence of Biomedical Science and Technology, Yangsan, Korea. ${ }^{14}$ Department of Neurology, Konyang University College of Medicine, Daejeon, Korea. ${ }^{15}$ Department of Neurology, Yonsei University Wonju College of Medicine, Wonju, Korea. ${ }^{16}$ Department of Neurology, Keimyung University Daegu Dongsan Hospital, Daegu, Korea. ${ }^{17}$ Department of Health Sciences and Technology, SAIHST, Sungkyunkwan University, Seoul, Korea. ${ }^{18}$ Stem Cell \& Regenerative Medicine Institute, Samsung Medical Center, Seoul, Korea. ${ }^{19}$ Department of Clinical Research Design and Evaluation, SAIHST, Sungkyunkwan University, Seoul, Korea. ${ }^{20}$ Center for Clinical Epidemiology, Samsung Medical Center, Seoul, Korea. ${ }^{21}$ Department of Neurology, Inha University School of Medicine, Incheon, Korea.

Received: 27 August 2019 Accepted: 23 December 2019

Published online: 04 January 2020

\section{References}

1. McKhann GM, Knopman DS, Chertkow H, Hyman BT, Jack CR Jr, Kawas CH, et al. The diagnosis of dementia due to Alzheimer's disease: recommendations from the National Institute on Aging-Alzheimer's Association workgroups on diagnostic guidelines for Alzheimer's disease. Alzheimers Dement. 2011;7:263-9.

2. Jack CR Jr, Albert MS, Knopman DS, McKhann GM, Sperling RA, Carrillo MC, et al. Introduction to the recommendations from the National Institute on Aging-Alzheimer's Association workgroups on diagnostic guidelines for Alzheimer's disease. Alzheimers Dement. 2011;7:257-62.

3. Hong YJ, Park KW, Kang DY, Lee JH. Prediction of Alzheimer's pathological changes in subjective cognitive decline using the self-report questionnaire and neuroimaging biomarkers. Dement Neurocogn Disord. 2019;18:19-29.

4. Thurfjell L, Lilja J, Lundqvist R, Buckley C, Smith A, Vandenberghe R, et al. Automated quantification of 18F-flutemetamol PET activity for categorizing scans as negative or positive for brain amyloid: concordance with visual image reads. J Nucl Med. 2014;55:1623-8.

5. Villemagne VL, Pike KE, Chetelat G, Ellis KA, Mulligan RS, Bourgeat P, et al. Longitudinal assessment of Abeta and cognition in aging and Alzheimer disease. Ann Neurol. 2011;69:181-92.

6. Rosenberg PB, Wong DF, Edell SL, Ross JS, Joshi AD, Brasic JR, et al. Cognition and amyloid load in Alzheimer disease imaged with florbetapir F 18(AV-45) positron emission tomography. Am J Geriatr Psychiatry. 2013;21:272-8.

7. Barthel H, Gertz HJ, Dresel S, Peters O, Bartenstein P, Buerger K, et al. Cerebral amyloid-beta PET with florbetaben (18F) in patients with Alzheimer's disease and healthy controls: a multicentre phase 2 diagnostic study. Lancet Neurol. 2011;10:424-35.

8. Vos SJB, van Boxtel MPJ, Schiepers OJG, Deckers K, de Vugt M, Carriere I, et al. Modifiable risk factors for prevention of dementia in midlife, late life and the oldest-old: validation of the LIBRA index. J Alzheimers Dis. 2017:58:537-47.

9. Thal DR, Rub U, Orantes M, Braak H. Phases of a beta-deposition in the human brain and its relevance for the development of AD. Neurology. 2002;58:1791-800.

10. Choi SH, Lee BH, Kim S, Hahm DS, Jeong JH, Yoon SJ, et al. Interchanging scores between clinical dementia rating scale and global deterioration scale. Alzheimer Dis Assoc Disord. 2003;17:98-105.

11. Petersen RC, Smith GE, Waring SC, Ivnik RJ, Tangalos EG, Kokmen E. Mild cognitive impairment: clinical characterization and outcome. Arch Neurol. 1999:56:303-8.

12. Diagnostic and Statistical Manual of Mental Disorders: DSM-IV-TR. Washington, DC, USA: American Psychiatric Association; 2000.

13. Hwang J, Jeong JH, Yoon SJ, Park KW, Kim EJ, Yoon B, et al. Clinical and biomarker characteristics according to clinical spectrum of Alzheimer's disease (AD) in the validation cohort of Korean brain aging study for the early diagnosis and prediction of AD. J Clin Med. 2019;8:341-59.

14. Farrar $\mathrm{G}$. Regional visual read inspection of [18F] flutemetamol brain images from end-of-life and amnestic MCl subjects. J Nucl Med. 2017;58:1250.

15. Martínez G, Flicker L, Vernooij RWM, Padilla PF, Zamora J, Figuls MR i, et al. 18F PET ligands for the early diagnosis of Alzheimer's disease dementia and 
other dementias in people with mild cognitive impairment (MCl). Cochrane Database Syst Rev. 2016. https://doi.org/10.1002/14651858.CD012216.

16. Olsson A, Vanderstichele $H$, Andreasen N, De Meyer G, Wallin A, Holmberg $B$, et al. Simultaneous measurement of beta-amyloid (1-42), total tau, and phosphorylated tau (Thr181) in cerebrospinal fluid by the XMAP technology. Clin Chem. 2005;51:336-45.

17. Shaw LM, Vanderstichele H, Knapik-Czajka M, Clark CM, Aisen PS, Petersen $\mathrm{RC}$, et al. Cerebrospinal fluid biomarker signature in Alzheimer's disease neuroimaging initiative subjects. Ann Neurol. 2009;65:403-13.

18. Kang JH, Korecka M, Toledo JB, Trojanowski JQ, Shaw LM. Clinical utility and analytical challenges in measurement of cerebrospinal fluid amyloid-beta (1-42) and tau proteins as Alzheimer disease biomarkers. Clin Chem. 2013;59:903-16.

19. Manu Vandijck RK, Waligorska T, De Smet S, Tsukie T, Verdoodt L, Deleersnijder W, Trojanowski J, Iwatsubo T, Shaw L. Inter-laboratory variation when using a unified test procedure for INNO-BIA AlzBio3. Alzheimer Dement. 2013;9:209-10.

20. Shaw LM, Vanderstichele H, Knapik-Czajka M, Figurski M, Coart E, Blennow K, et al. Qualification of the analytical and clinical performance of CSF biomarker analyses in ADNI. Acta Neuropathol. 2011;121:597-609.

21. Lee JH, Lee KU, Lee DY, Kim KW, Jhoo JH, Kim JH, et al. Development of the Korean version of the consortium to establish a registry for Alzheimer's disease assessment packet (CERAD-K): clinical and neuropsychological assessment batteries. J Gerontol B Psychol Sci Soc Sci. 2002;57:P47-53.

22. Ahn HJ, Chin J, Park A, Lee BH, Suh MK, Seo SW, et al. Seoul neuropsychological screening battery-dementia version (SNSB-D): a useful tool for assessing and monitoring cognitive impairments in dementia patients. J Korean Med Sci. 2010;25:1071-6.

23. Kang SH, Park YH, Lee D, Kim JP, Chin J, Ahn Y, et al. The cortical Neuroanatomy related to specific neuropsychological deficits in Alzheimer's continuum. Dement Neurocogn Disord. 2019;18:77-95.

24. Kim HJ, Park S, Cho H, Jang YK, San Lee J, Jang H, et al. Assessment of extent and role of tau in subcortical vascular cognitive impairment using 18F-AV1451 positron emission tomography imaging. JAMA Neurol. 2018:75:999-1007.

25. Cho H, Choi JY, Hwang MS, Kim YJ, Lee HM, Lee HS, et al. In vivo cortical spreading pattern of tau and amyloid in the Alzheimer disease spectrum. Ann Neurol. 2016;80:247-58.

26. Mormino EC, Kluth JT, Madison CM, Rabinovici GD, Baker SL, Miller BL, et al. Episodic memory loss is related to hippocampal-mediated beta-amyloid deposition in elderly subjects. Brain. 2009;132:1310-23.

27. Perrotin A, Mormino EC, Madison CM, Hayenga AO, Jagust WJ. Subjective cognition and amyloid deposition imaging: a Pittsburgh compound B positron emission tomography study in normal elderly individuals. Arch Neurol. 2012;69:223-9.

28. Pike KE, Savage G, Villemagne VL, Ng S, Moss SA, Maruff P, et al. Betaamyloid imaging and memory in non-demented individuals: evidence for preclinical Alzheimer's disease. Brain. 2007:130:2837-44.

29. Huang KL, Lin KJ, Hsiao IT, Kuo HC, Hsu WC, Chuang WL, et al. Regional amyloid deposition in amnestic mild cognitive impairment and Alzheimer's disease evaluated by [18F]AV-45 positron emission tomography in Chinese population. PLoS One. 2013:8:e58974.

30. Duff K, Foster NL, Dennett K, Hammers DB, Zollinger LV, Christian PE, et al. Amyloid deposition and cognition in older adults: the effects of premorbid intellect. Arch Clin Neuropsychol. 2013;28:665-71.

31. Landau SM, Lu M, Joshi AD, Pontecorvo M, Mintun MA, Trojanowski JQ, et al. Comparing positron emission tomography imaging and cerebrospinal fluid measurements of beta-amyloid. Ann Neurol. 2013;74:826-36.

32. Schipke CG, Koglin N, Bullich S, Joachim LK, Haas B, Seibyl J, et al. Correlation of florbetaben PET imaging and the amyloid peptide Ass 42 in cerebrospinal fluid. Psychiatry Res Neuroimaging. 2017;265:98-101.

33. Cho SH, Shin JH, Jang H, Park S, Kim HJ, Kim SE, et al. Amyloid involvement in subcortical regions predicts cognitive decline. Eur J Nucl Med Mol Imaging. 2018:45:2368-76.

34. Grothe MJ, Barthel H, Sepulcre J, Dyrba M, Sabri O, Teipel SJ, et al. In vivo staging of regional amyloid deposition. Neurology. 2017;89:2031-8.

35. Hanseeuw BJ, Betensky RA, Mormino EC, Schultz AP, Sepulcre J, Becker JA, et al. PET staging of amyloidosis using striatum. Alzheimers Dement. 2018; 14:1281-92

\section{Publisher's Note}

Springer Nature remains neutral with regard to jurisdictional claims in published maps and institutional affiliations.

\section{Ready to submit your research? Choose BMC and benefit from:}

- fast, convenient online submission

- thorough peer review by experienced researchers in your field

- rapid publication on acceptance

- support for research data, including large and complex data types

- gold Open Access which fosters wider collaboration and increased citations

- maximum visibility for your research: over $100 \mathrm{M}$ website views per year

At BMC, research is always in progress.

Learn more biomedcentral.com/submissions 